\title{
DISCOVERY OF A HALO AROUND THE HELIX NEBULA NGC 7293 IN THE WISE ALL-SKY SURVEY
}

\author{
Yong Zhang, Chin-Hao Hsia, and Sun KwoK \\ Department of Physics, Faculty of Science, The University of Hong Kong, Hong Kong, China; zhangy96@hku.hk, xiazh@hku.hk, sunkwok@hku.hk \\ Received 2012 April 10; accepted 2012 June 6; published 2012 July 25
}

\begin{abstract}
We report the discovery of an extended halo $\left(\sim 40^{\prime}\right.$ in diameter) around the planetary nebula NGC 7293 (the Helix Nebula) observed in the $12 \mu \mathrm{m}$ band from the Wide-field Infrared Survey Explorer all-sky survey. The mid-infrared halo has an axisymmetric structure with a sharp boundary to the northeast and a more diffuse boundary to the southwest, suggesting an interaction between the stellar wind and the interstellar medium (ISM). The symmetry axis of the halo is well aligned with that of a northeast arc, suggesting that the two structures are physically associated. We have attempted to fit the observed geometry with a model of a moving steady-state stellar wind interacting with the ISM. Possible combinations of the ISM density and the stellar velocity are derived from these fittings. The discrepancies between the model and the observations suggest that the stellar mass loss has a more complicated history, including possible time and angle dependences.
\end{abstract}

Key words: infrared: ISM - ISM: structure - planetary nebulae: individual: Helix Nebula (NGC 7293) - stars: AGB and post-AGB

Online-only material: color figures

\section{INTRODUCTION}

As low- and intermediate-mass stars evolve from asymptotic giant branch (AGB) to planetary nebula (PN) stages, they continually eject matter into the interstellar medium (ISM). The resultant circumstellar envelopes are one of the main sources of chemical enrichment of the Galaxy. Our current understanding of the origin of PNs is based on the interacting stellar wind (ISW) model (Kwok 1982) which has predicted the presence of AGB halos created by AGB winds. The remnants of this AGB wind are swept up by a later-developed fast wind to form a PN. One of the predictions of the model is that the remnants of the AGB wind should be detectable as halos in PNs. Since then, AGB halos have been detected by a variety of techniques. These include observations (1) in the visible through emission or scattering, (2) in millimeter and submillimeter wavelengths through molecular-line emission, and (3) in the infrared through dust emission. Since PN halos can contain more mass than the main bright nebular shells, studies of PN halos are important to understand the AGB mass-loss history and the chemical evolution of the Galaxy.

In the visible, AGB halos are usually very faint (with surface brightness about $10^{-3}-10^{-4}$ lower than the main nebulae), making them difficult to observe. Many efforts have been made to search for faint AGB halos (see e.g., Corradi et al. 2003; Ramos-Larios \& Phillips 2009). These observations show that the detected AGB halos usually exhibit a bow-shaped bright rim, suggesting the interaction between AGB winds and the ISM. The wind-ISM interaction process has been the subject of various theoretical studies (see e.g., Smith 1976; Borkowski et al. 1990; Soker et al. 1991; Dgani \& Soker 1998; Villaver et al. 2003; Wareing et al. 2007a, 2007b). Early studies suggest that the wind-ISM interaction is observable only for evolved PNs whose density has dropped below a critical limit. More recent hydrodynamic simulations argue that the interaction can be observed for young PNs if the time-dependent variations in AGB and post-AGB winds are taken into account. The wind-ISM interaction was indeed observed in not only ancient PNs (Tweedy \& Kwitter 1996) but also in the circumstellar envelopes of AGB stars (Ueta et al. 2006; Ueta 2008; Cox et al. 2012).
The high-quality images acquired by recent wide field-ofview (FOV) surveys have been proven useful to search for largescale extended structures around evolved stars. For example, Sahai \& Chronopoulos (2010) discovered a large outer shell around the highly evolved AGB star IRC+10216 using the images taken with Galaxy Evolution Explorer (GALEX; Martin et al. 2005). In this paper, we report the results of searching for outer structures around NGC 7293 utilizing the recently released mid-infrared images of the Wide-field Infrared Survey Explorer (WISE; Wright et al. 2010) all-sky survey.

NGC 7293 is one of the most extensively studied PNs (see O'Dell et al. 2004; Hora et al. 2006; Speck et al. 2002; Meaburn et al. 1992, 2005, 2008 and reference therein). Given its relative proximity to the Sun and its relatively accurate distance measurement, NGC 7293 is an excellent target to study in detail the spatial structure of PNs. In this paper, we have adopted the distance estimate of $216_{-12}^{+14} \mathrm{pc}$ as determined by Benedict et al. (2009). Although its overall appearance seems simple, a number of intriguing structures, from small cometary knots to large-scale arcs, to bipolar outflows, have been revealed in previous observations. The inner helical structure is composed of thousands of cometary knots of lowly ionized and molecular gas. The main nebula consists of two rings of highly ionized gas and a faint outer filament. The three-dimensional (3D) structure of the main nebula has been investigated by Meaburn et al. (2008) and O'Dell et al. (2004), who suggested that it was formed through multiple outflows along different directions.

Although NGC 7293 is of great astrophysical interest, it is not an easy object to observe because of its low surface brightness and its large angular extent in the sky. Without highly sensitive and large FOV observations, it is impossible to explore the faint halo around this PN. The recent WISE observations, therefore, provide an invaluable resource for that purpose.

\section{THE DATA}

The data used in this paper were taken from the WISE All-Sky Data Release ${ }^{1}$. The data have been calibrated with the WISEpipeline. WISE mapped the entire sky in four bands centered at

\footnotetext{
1 See http://wise2.ipac.caltech.edu/docs/release/allsky/.
} 

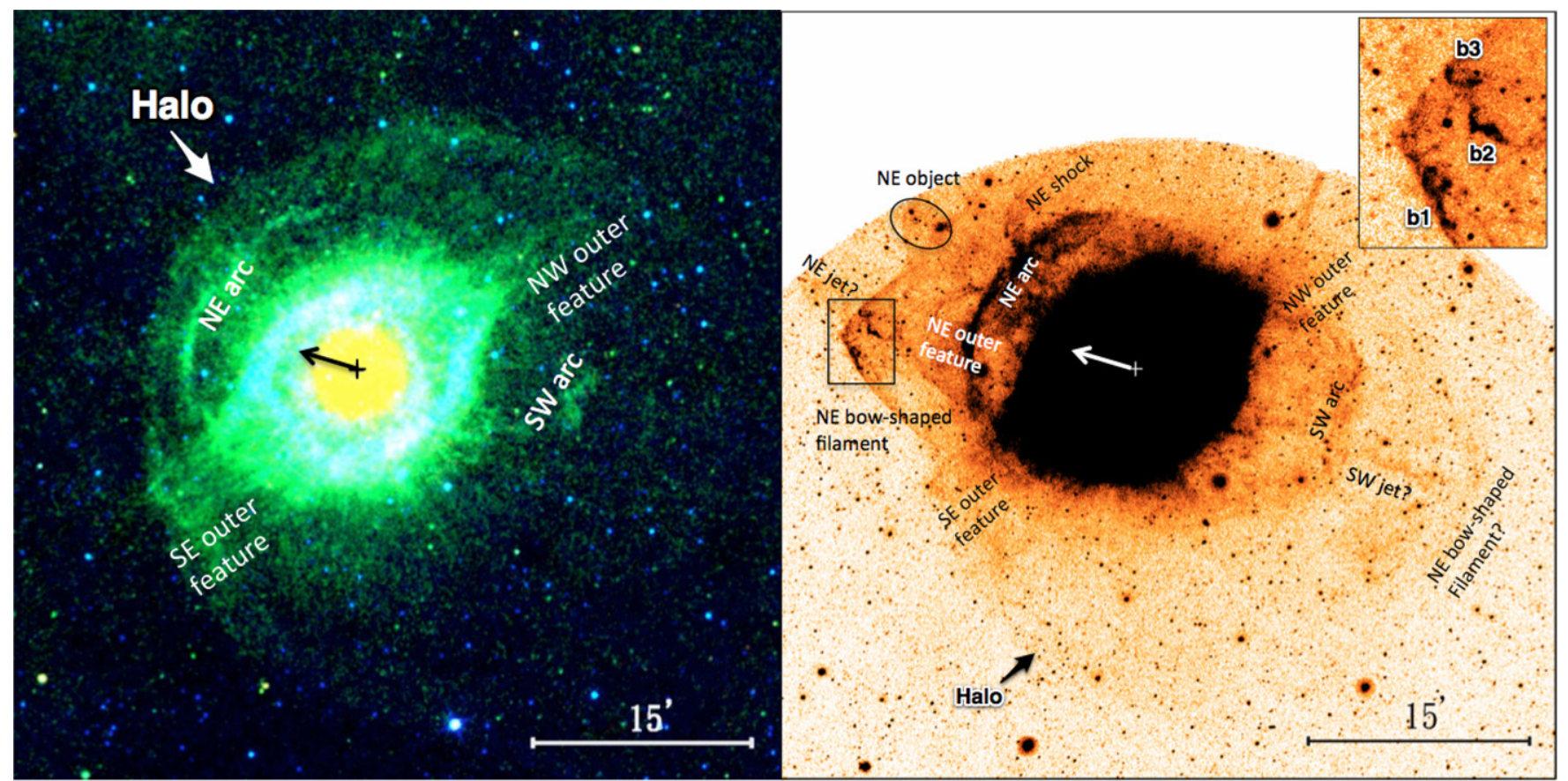

Figure 1. Composite-color WISE image (left panel) and false-color GALEX image (right panel) of NGC 7293. Left is east, and up is north. In the left panel, the WISE $3.4 \mu \mathrm{m}$ band is assigned the color blue, the $12 \mu \mathrm{m}$ band the green, and the $22 \mu \mathrm{m}$ band the red. The labels "NW outer feature" and "SE outer feature" are the terms assigned by O'Dell et al. (2004) for the bipolar outflow. In the right panel, the top-right corner panel shows a zoomed-in view of the black box. The cross and arrow at the center of each panel represent the location of the central star and the direction of proper motion. An axisymmetric halo is clearly visible in the WISE image. Note that in the GALEX image, the spike above the NW outer feature is artificial.

(A color version of this figure is available in the online journal.)

wavelengths of $3.4,4.6,12$, and $22 \mu \mathrm{m}$ (W1-W4) with angular resolutions of 6". 1,6 .'4, 6". 5 , and $12^{\prime \prime} 0$, respectively. During the survey, the four bands were simultaneously deployed to image a $47^{\prime} \times 47^{\prime} \mathrm{FOV}$ on the sky. The exposure time of an individual frame is $7.7 \mathrm{~s}$ in $\mathrm{W} 1$ and $\mathrm{W} 2$, and $8.8 \mathrm{~s}$ in $\mathrm{W} 3$ and $\mathrm{W} 4$. The number of exposures are different at different ecliptic latitudes. The observations of the fields including NGC 7293 were made on 2010 May 20-23, with a total accumulated time of 17.2 minutes for $\mathrm{W} 1$ and $\mathrm{W} 2$, and 19.7 minutes for $\mathrm{W} 3$ and $\mathrm{W} 4$. The composite-color WISE image of NGC 7293 is shown in Figure 1.

Our analysis is supplemented by the far- and near-ultraviolet (FUV and NUV) images retrieved from the GALEX archive. The FUV and NUV images have effective wavelengths of $1516 \AA$ and $2267 \AA$, and angular resolutions of 4.5 and $6^{\prime \prime}$, respectively. A detailed description of the calibration and data products of GALEX can be found in Morrissey et al. (2007). The observations of NGC 7293 were made on 2004 August 22-25 as part of the Calibration Imaging Survey (CAI). The accumulated exposure time is 56 minutes for each band. In order to improve the signal-to-noise ratio, we co-added the FUV and NUV images. The resultant GALEX image is shown in the right panel of Figure 1.

\section{RESULTS}

An axisymmetric extended halo at $12 \mu \mathrm{m}$ surrounding NGC 7293 can clearly be seen in Figure 1. The halo exhibits a bow-like shape with a well-defined boundary to the northeast (NE), in contrast to a diffuse and open structures to the southwest (SW). Such asymmetric morphology gives a clear indication that the object is moving toward the NE. The direction of motion can be approximated by the symmetry axis of the halo and the symmetry axis is found to be located at a position angle (PA) of $60^{\circ}$. The NE boundary of the halo can be approximately described by a circle with a radius of $1213^{\prime \prime}$ $\left(3.9 \times 10^{13} \mathrm{~km}=2.6 \times 10^{5} \mathrm{AU}=1.3 \mathrm{pc}\right)$, with a center located at $359^{\prime \prime}\left(1.2 \times 10^{13} \mathrm{~km}=7.8 \times 10^{4} \mathrm{AU}=0.4 \mathrm{pc}\right)$ from the central star (see Figure 2).

We find that the $12 \mu \mathrm{m}$ emission in the halo is relatively smooth and is about 10 times fainter than that of the main nebula. This halo is not detectable at the WISE $22 \mu \mathrm{m}$ band and has not been seen in previously reported optical images. It is only marginally detectable in the WISE 3.4 and $4.6 \mu \mathrm{m}$ and in the GALEX images. Assuming that the infrared emission peaks at $12 \mu \mathrm{m}$, we infer that the emission of the halo is dominated by thermal emission from warm dust with a temperature of $\sim 300 \mathrm{~K}$. One can, in principle, obtain the spatial distribution of temperature in the halo by comparing the W1-W3 images. However, the halos in W1 and W2 are too faint to yield meaningful conclusions. Nevertheless, we cannot rule out the possibility that shock-excited $\mathrm{H}_{2}$ lines might significantly contribute to the midinfrared emission of the halo, as suggested by Ramos-Larios et al. (2010) in their study of the halo of NGC 40.

The shape of the halo obviously suggests that it is interacting with the ISM. The conclusion is strengthened by the study of the proper motion of the Helix Nebula by Kerber et al. (2008). The direction of the proper motion is found to be at $\mathrm{PA}=77^{\circ}$, which roughly aligns with the symmetry axis of the halo and the bow apex. The apex of the bow-shaped halo (position $A$ in Figure 2) is about $854^{\prime \prime}\left(2.8 \times 10^{13} \mathrm{~km}=1.8 \times 10^{5} \mathrm{AU}=0.9 \mathrm{pc}\right)$ from the central star. Assuming the velocity of the AGB wind, $v_{\mathrm{w}}=10 \mathrm{~km} \mathrm{~s}^{-1}$, we obtain a lower limit to the dynamic age of the halo of $88,000 \mathrm{yr}$, suggesting that the halo was indeed formed long ago, and can therefore serve as a fossil record of the AGB mass-loss history. O'Dell et al. (2004) estimated that 


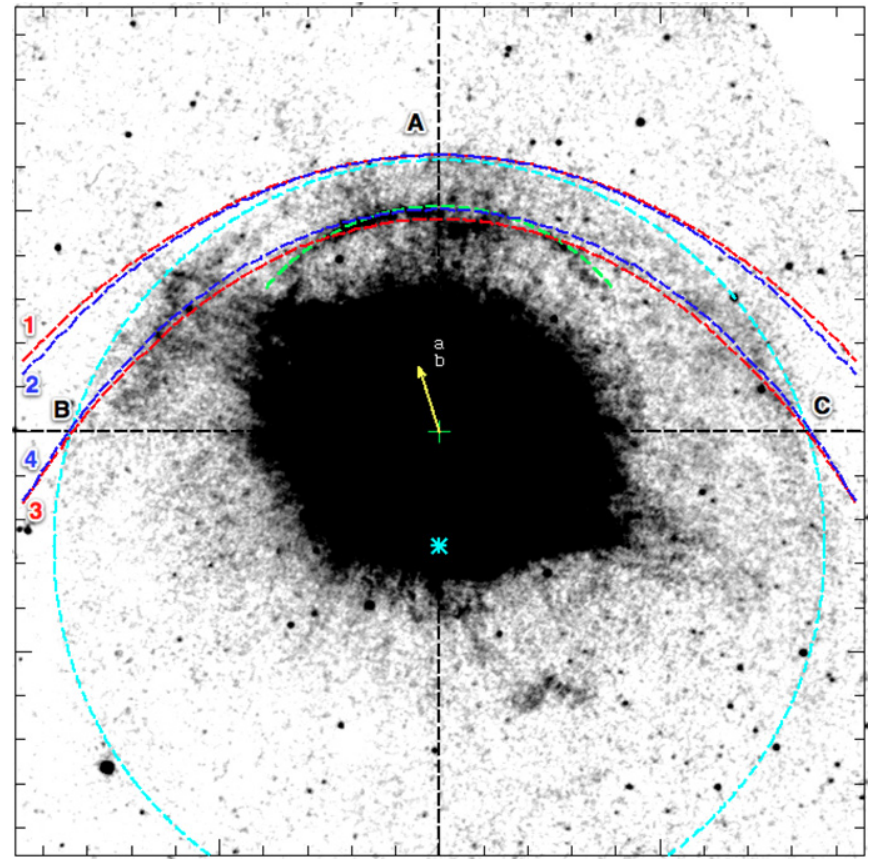

Figure 2. WISE $12 \mu \mathrm{m}$ image shown in gray scale. The area of the image is $44^{\prime} \times 44^{\prime}$, with the north direction $60^{\circ}$ clockwise from the vertical axis. The cross and arrow represent the location of the central star and the direction of proper motion (as in Figure 1). The boundary of the halo is indicated by the cyan dashed circle with a radius of $20^{\prime}$ from its center (denoted by a cyan colored asterisk). The position of the NE arc is indicated by the green dashed circular arc with a radius of 11.7 from the central star. The red and blue dashed curves represent the four models listed in Table 1, and the number of the model is labeled next to the curve. The projected locations of the apexes of models 2 and 4 are marked by labels "a" and "b."

(A color version of this figure is available in the online journal.)

the outer material of NGC 7293 has a recombination timescale of $\sim 40,000 \mathrm{yr}$, much shorter than the dynamic age of the halo. Therefore, it is very likely that the halo is neutral and optically invisible. Based on optical observations, Meaburn et al. (2008) determined the dynamic age of the main nebula to be 11,000 yr. If the halo has been continually developed with a mass-loss rate of $\dot{M}=10^{-5} M_{\odot} \mathrm{yr}^{-1}$ and was terminated when a fast wind was launched to form the main nebula, the mass of the halo can be estimated to be $>0.77 M_{\odot}$. Due to the wind-ISM interaction, the past of the halo material has probably been stripped to the SW behind the central star.

The WISE images also reveal some smaller-scale structures such as the NE arc and small cometary knots. The main nebula exhibits a collimated bipolar outflow oriented NW-SE (the NW and SE outer features in Figure 1, as assigned by O'Dell et al. 2004). We note that the SE ends of the bipolar outflow are running into the boundary of the halo. These results are consistent with the narrower FOV infrared observations acquired with the Spitzer Space Telescope by Hora et al. (2006). The NE arc is the brightest feature outside the main nebula. Figure 2 shows that the $12 \mu \mathrm{m}$ image of the NE arc can be described by a circle arc about 696" from the central star while its FUV emission is closer to the central star (about 680" away). Hora et al. (2006) found that the $\mathrm{H}_{2}$ mid-infrared emission of the $\mathrm{NE}$ arc is located in a shell outside the $\mathrm{H} \alpha$ emission. Because the GALEX FUV emission is presumably from shock-excited $\mathrm{H}_{2}$, the stratified optical/FUV and mid-infrared emission structures likely suggest the radial variations of excitation conditions rather than the $\mathrm{H}^{+} / \mathrm{H}_{2}$ ratios. We also note that the WISE $22 \mu \mathrm{m}$ image shows strong brightening around the central star. This confirms the previous Spitzer observations at $24 \mu \mathrm{m}$ by Su et al. (2007) who ascribed the $24 \mu \mathrm{m}$ brightening to the presence of a inner debris disk.

In the outer region, the GALEX and WISE images appear strikingly different (see Figure 1). The GALEX image shows many bow-shaped features that are not detected by WISE. A schematic presentation of the observed structures is presented in Figure 6 of Meaburn et al. (2008). These FUV features are presumably due to $\mathrm{H}_{2}$ emission excited by fast shock waves. The most interesting feature is the NE bow-shaped filament, which contains three bow-shaped sub-structures $(b 1-b 3$ in the inset of Figure 1) and whose apex is exactly aligned with the direction of proper motion (see the proper motion vector in Figure 1). These sub-structures were probably formed due to instabilities in the shock interface, as predicted by the simulations of Mohamed et al. (2012). We note that the apex of the fast outflow shown in the GALEX image has an " $L$ " shape, which is characteristic of an outflow near the plane of the sky. If the outflow is inclined at a large angle, then the apex would take on a more rounded shape.

Unlike the bow-shaped filament, the NE boundary of the halo cannot be seen in the GALEX image. This is probably the result of the shock responsible for the halo boundary being too slow to provide enough energy to excite $\mathrm{H}_{2}$ emission. The GALEX image also shows the NE object and shock (see O'Dell et al. 2004, for a discussion), as well as bipolar streams in NE-SW orientation (marked as "outer features" in Figure 1). Although there are several asymmetrical outflows (outside and inside the halo), the halo boundary seems to be continuous and not broken (as seen in the left panel of Figure 1). This simply suggests that from the perspective perpendicular to the line of sight, these streams do not cross the projected halo boundary.

The mid-infrared halo has an appearance similar to the shell around IRC+10216 detected by Sahai \& Chronopoulos (2010), except that the vortices behind the IRC+10216 shell are not clearly detected in NGC 7293. If the vortices had been formed behind the halo of NGC 7293, then they supposedly would have been diluted to the ISM.

Overall, the newly discovered mid-infrared halo of the well studied PN NGC 7293 strengthens the conclusions that AGB halos are commonly present and infrared imaging is particularly useful to detect the faint extended structures (e.g., Ramos-Larios \& Phillips 2009; Ramos-Larios et al. 2012). The direct detection of the halo of NGC 7293 provides us with the knowledge of the ambient environment within which the later-developed dynamical processes (e.g., the multipolar fast outflows, Meaburn et al. 2008 operate. By deriving the geometry and density of the halo, we are in a much better position to constrain the physical processes at work.

\section{DISCUSSION}

The shape of the $12 \mu \mathrm{m}$ halo suggests the presence of a bow shock. Since the shape of bow shock is dependent on the velocity and density contrasts between the matter of the stellar wind and the ISM, the observed structure can serve as a useful probe of the properties of the AGB wind and the ISM. If the post-shock material has a high cooling rate, then the physical thickness of the shell at the shock interface would be small. Under the thinshell approximation and assuming that an equilibrium between the ram pressure of stellar wind and that of the ISM is reached, Wilkin (1996) deduced an analytic expression for the shape of 
Table 1

The Modeling Results ${ }^{\mathrm{a}}$

\begin{tabular}{lccc}
\hline \hline Model & $i$ & $\begin{array}{c}R_{0} \\
\left(10^{18} \mathrm{~cm}\right)\end{array}$ & $\begin{array}{c}v_{*} / \sqrt{n_{\text {ISM }}} \\
\left(\mathrm{km} \mathrm{s}^{-1} \mathrm{~cm}^{1.5}\right)\end{array}$ \\
\hline 1 & $\left({ }^{\circ}\right)$ & 2.78 & 17.2 \\
2 & 0 & 1.80 & 26.5 \\
3 & 60 & 2.13 & 22.3 \\
4 & 0 & 1.44 & 33.0 \\
\hline
\end{tabular}

Note. ${ }^{\text {a }}$ Assuming $\dot{M}=10^{-5} M_{\odot} \mathrm{yr}^{-1}, v_{\mathrm{w}}=10 \mathrm{~km} \mathrm{~s}^{-1}$, and the dimensionless mean molecular weight of 1.33 .

the bow shock,

$$
R(\theta)=R_{0} \sin ^{-1} \theta \sqrt{3(1-\theta \cot \theta)},
$$

where $\theta$ is the polar angle from the symmetric axis as seen from the central star at the coordinate origin, and $R_{0}$ is the distance between the apex and the central star (i.e., stand-off distance) given by

$$
R_{0}=\sqrt{\dot{M} v_{\mathrm{w}} /\left(4 \pi \rho_{\mathrm{ISM}} v_{*}^{2}\right)}
$$

In Equation (2), $\rho_{\mathrm{ISM}}$ is the mass density of the ISM and $v_{*}$ the velocity of the star in respect to the ISM. $R(\theta)$ increases with increasing $\theta$ because the ram pressure of the ISM is the strongest at the apex and decreases on either side. Assuming that the properties of the stellar wind are typical (see Table 1), one can obtain the relation between $v_{*}$ and the number density ( $\left.n_{\text {ISM }}\right)$ of the ISM from Equation (2) by measuring $R_{0}$. Wilkin's analytic solutions have been widely applied to study the bow shocks around a variety of objects including young stellar objects, red supergiants, AGB stars, and pulsars (e.g., Cox et al. 2012; MacKey et al. 2012; Jorissen et al. 2011).

We have attempted to determine the $R_{0}$ value by fitting the projected shape of a 3D paraboloid (constructed from Equation (1)) on the sky plane with the observations. For given assumed values of $R_{0}$ and $i$ (inclination angle of the symmetric axis in respect to the sky plane, no matter whether the apex points toward or away from us), the projected distances between every point on the paraboloid and the central star can be determined. The boundary of the projected 3D paraboloid is found by calculating the furthermost projected distance at each position angle in the sky plane. Values of $R_{0}$ and $i$ are then adjusted to obtain the best fit. Similar procedures have been used by Cox et al. (2012) to study the bow shocks around AGB stars and red supergiants. We should note that since the bow shock has a sharp boundary, the inclination angle cannot be too large (i.e., the star is moving not far from the plane of the sky).

We constructed a series of models with different $i$ and $R_{0}$ values, but found none of them can completely account for the shape of the halo boundary. Figure 2 shows four of the models, and the model parameters are listed in Table 1 . We can see that as $i$ increases, the projected location of the apex of the paraboloid changes from $A$ toward the central star (positions $a$ and $b$ ). If $i$ were $90^{\circ}$, the projected position of the apex would be at the central star. The models suggest that given a certain distance from the projected apex (position $A$ ) to the central star, the values of $R_{0}$ monotonically decreases with increasing $i$ (see models 1 and 2). This is consistent with the results of Mac Low et al. (1991) and Cox et al. (2012). Since the shapes of the model curves are insensitive to $i$, we are unable to match the observed positions of $B$ and $C$. If we force the model curves to pass through $B$ and $C$ (see models 3 and 4), then the model boundary curves deviate from the position of $A$. Nevertheless, our models suggest that the $v_{*} / \sqrt{n_{\mathrm{ISM}}}$ value is probably in the range of $15-50 \mathrm{~km} \mathrm{~s}^{-1} \mathrm{~cm}^{1.5}$. For example, in model 1, the number density of $\mathrm{H}$ in the ISM would be $0.3 \mathrm{~cm}^{-3}$ if the star is moving at a speed of $10 \mathrm{~km} \mathrm{~s}^{-1}$.

Below, we will discuss the possible causes for the inability of Wilkin's expression in fitting the observations. If the post-shock material is adiabatic, a termination shock and a bow shock are expected to form inside and outside the halo, respectively. In this case, the boundary of the halo is the discontinuity between stellar and interstellar material (i.e., astropause). These structures have been detected in the shell of IRC+10216 (Sahai \& Chronopoulos 2010). One may have an impression from Figure 1 that the NE bow-shaped filament detected by GALEX and the NE arc are located outside and inside the halo, respectively, and perhaps they represent the bow shock and the termination shock. This scenario, however, is unlikely because the surface brightnesses of the three components are significantly different from those observed in IRC+10216 and predictions of simulations. Moreover, the NE bow-shaped filament and arc have very faint counterparts on opposite sides of the central star (see the right panel of Figure 1), suggesting that they were likely developed through different ejection events. The appearance of the NE arc is similar to that of the brightening shell as predicted by the simulations of Wareing et al. (2007a), probably suggesting that it was formed by the interaction between a fast wind and the early AGB wind. Furthermore, the appearances of the NE arc and its SW counterpart suggest that it is likely to be a debris of the ring, a feature that has been frequently detected in halos of PNs (Corradi et al. 2004).

Equations (1) and (2) are steady-state solutions. In the simulations of Betelgeuse's bow shock, Mohamed et al. (2012) found that it takes a long time $(\geqslant 30,000 \mathrm{yr})$ for the bow shock to reach a steady state, before which the $R\left(90^{\circ}\right) / R\left(0^{\circ}\right)$ ratio increases with time from 1 to $\sqrt{3}$. Assuming that the symmetric axis of the halo of NGC 7293 lies in the sky plane (i.e., $i=0^{\circ}$ ), we obtain $R\left(90^{\circ}\right) / R\left(0^{\circ}\right)=1.32$. Therefore, a possible explanation for the mismatch in the model and observation results is that the halo has not reached a steady state even though it is very old.

In addition, Wilkin's solutions are based on the assumption of a constant stellar wind, which is applicable for the early AGB stage. However, as the star evolves up the AGB, both the mass-loss rate and wind velocity increase in magnitude. A jump in the stellar wind velocity in the post-AGB phase of evolution will cause interactions among stellar winds, which will also have an effect on the shape of the halo boundary. The shape of the halo can also be affected by additional mechanisms such as magnetic fields, which may distort the halo boundary. van Marle et al. (2011) performed simulations including drag force between dust and gas. They found that depending on sizes of dust grains, infrared observations of bow shocks may not represent the morphologies of gas. The most likely explanation of the discrepancy between the model and the observed halo morphology is a latitude dependence of the density distribution of the AGB wind. AGB winds are known to develop non-spherically symmetric outflows near the end of AGB evolution, and this can easily change the shape of the interaction region. This equatorial enhanced mass loss have been attributed to binary central stars, star-planet interaction, star spots, and other mechanisms, but the exact cause is yet to be identified. Another possible complication is the angular 
dependence of the later-developed fast wind (bipolar outflow), which can distort the density distribution of the AGB envelope. Further numerical simulations including multiple stellar winds will be needed. Additional variables such as time-dependence and angle-dependence of outflows need to be investigated. The observed halo morphology presented here will serve as useful constraints to these models.

Recently, Ramos-Larios et al. (2012) discovered an outer halo around the young PN IC 418 based on the WISE images. Unlike that of NGC 7293, the halo of IC 418 does not exhibit a cometary shape and has a well defined boundary, suggesting that it has not been striped. Its elliptical appearance indicates that the density and velocity AGB winds are indeed angle-dependent. Villaver et al. (2003) performed 2-D numerical simulations of the PN-ISM interaction considering variable AGB mass-loss rates. The shape of the NGC 7293 halo is qualitatively similar to their results. The subsequent 3D simulations by Wareing et al. (2007a) predicted four stages of the PN-ISM interaction. According to their classification scheme, this evolved PN is in the first stage when the main nebula is unaffected by the interaction. Wareing et al. (2007b) showed that the Kelvin-Helmholtz instabilities at the shock front can lead to vortices in the wakes of AGB stars. Although we do not see unambiguous vortex-like structures in the halo of NGC 7293, the "SW arc" seemingly resembles the vortices reproduced by the models of Wareing et al. (2007b). Through theoretical analysis, Dgani \& Soker (1998) found that because of the Rayleigh-Taylor instability, the PN halo can be fragmented by the flow of ISM material. This phenomenon has been discovered in the PN NGC 6772 (RamosLarios \& Phillips 2009). We note that the halo appearance of NGC 7293 highly resembles that of NGC 6772. Both show a bright rimmed region in the shock head, fan-like material distribution in the tail, and ray-like enhancements in the interior. It follows that the Rayleigh-Taylor disruption is also present in the halo of NGC 7293. NGC 7293 is presumably more evolved than those PNs with halos investigated by Ramos-Larios \& Phillips (2009). We do not find tight relations between the properties of the halos and the ages of their host PNs. This should be ascribed to different environments surrounding these PNs which play an important role in the PN-ISM interaction processes.

\section{CONCLUSIONS}

From the imaging data of the WISE all-sky survey, we discover a mid-infrared halo with a diameter of $\sim 40^{\prime}$ around NGC 7293. The halo is totally invisible at optical wavelengths and emits light with a peak intensity near $12 \mu \mathrm{m}$. The morphology of the halo suggests that it has been shaped by wind-ISM interaction, and the NE arc is brightened through wind-wind interaction. We find that the shape of the halo boundary cannot be explained by the analytic solutions of Wilkin (1996) under the thin shock-shell and steady-state approximations. This suggests that mass loss during the progenitor AGB phase has a more complicated history due to time- and/or angular-dependent outflows.
PN systems are the results of dynamical interactions between the halo (remnants of AGB wind) with the interstellar medium, as well as the interactions between fast outflows with the remnants of the AGB wind (interacting winds). The quantitative information derived from this study is useful for the understanding of both types of dynamical interactions. NGC 7293, being the PN closest to us, is a valuable laboratory for the study of dynamic processes at work in the astrophysical environment.

We thank Sze Ning Chong for helpful discussions. We also thank the anonymous referee for helpful comments. This paper makes use of data products from the Wide-field Infrared Survey Explorer, which is a joint project of the University of California, Los Angeles, and the Jet Propulsion Laboratory/California Institute of Technology, funded by the National Aeronautics and Space Administration. Support for this work was provided by the Research Grants Council of the Hong Kong under grants HKU7031/10P and the Seed Funding Programme for Basic Research in HKU (200909159007).

\section{REFERENCES}

Benedict, G. F., McArthur, B. E., Napiwotzki, R., et al. 2009, AJ, 138, 1969 Borkowski, K. J., Sarazin, C. L., \& Soker, N. 1990, ApJ, 360, 173

Corradi, R. L. M., Sánchez-Blázquez, P., Mellema, G., Giammanco, C., \& Schwarz, H. E. 2004, A\&A, 417, 637

Corradi, R. L. M., Schönberner, D., Steffen, M., \& Perinotto, M. 2003, MNRAS, 340,417

Cox, N. L. J., Kerschbaum, F., van Marle, A.-J., et al. 2012, A\&A, 537, 35

Dgani, R., \& Soker, N. 1998, ApJ, 495, 337

Hora, J. L., Latter, W. B., Smith, H. A., \& Marengo, M. 2006, ApJ, 652, 426

Jorissen, A., Mayer, A., van Eck, S., et al. 2011, A\&A, 532, 135

Kerber, F., Mignani, R. P., Smart, R. L., \& Wicenec, A. 2008, A\&A, 479, 155 Kwok, S. 1982, ApJ, 258, 280

Mac Low, M.-M., van Buren, D., Wood, D. O. S., \& Churchwell, E. 1991, ApJ, 369,395

MacKey, J., Mohamed, S., Neilson, H. R., Langer, N., \& Meyer, D. M. A. 2012, ApJ, 751, L10

Martin, D. C., Fanson, J., Schiminovich, D., et al. 2005, ApJ, 619, L1 Meaburn, J., Boumis, P., López, J. A., et al. 2005, MNRAS, 360, 963 Meaburn, J., López, J. A., \& Richer, M. G. 2008, MNRAS, 384, 497 Meaburn, J., Walsh, J. R., Clegg, R. E. S., et al. 1992, MNRAS, 255, 177 Mohamed, S., Mackey, J., \& Langer, N. 2012, A\&A, 541, 1

Morrissey, P., Conrow, T., Barlow, T. A., et al. 2007, ApJS, 173, 682 O’Dell, C. R., McCullough, P. R., \& Meixner, M. 2004, AJ, 128, 2339 Ramos-Larios, G., \& Phillips, J. P. 2009, MNRAS, 400, 575 Ramos-Larios, G., Phillips, J. P., \& Cuesta, L. C. 2010, MNRAS, 411, 1245 Ramos-Larios, G., Vazquez, R., Guerrero, M. A., et al. 2012, MNRAS, in press Sahai, R., \& Chronopoulos, C. K. 2010, ApJ, 711, L53

Smith, H. 1976, MNRAS, 175, 419

Soker, N., Borkowski, K. J., \& Sarazin, C. L. 1991, AJ, 102, 1381

Speck, A. K., Meixner, M., Fong, D., et al. 2002, AJ, 123, 346

Su, K. Y. L., Chu, Y.-H., Rieke, G. H., et al. 2007, ApJ, 657, L41

Tweedy, R. W., \& Kwitter, K. B. 1996, ApJS, 107, 255

Ueta, T. 2008, ApJ, 687, L33

Ueta, T., Speck, A. K., Stencel, R. E., et al. 2006, ApJ, 648, L39

van Marle, A. J., Meliani, Z., Kepens, R., \& Decin, L. 2011, ApJ, 734, L26

Villaver, E., García-Segura, G., \& Manchado, A. 2003, ApJ, 585, L49

Wareing, C. J., Zijlstra, A. A., \& O’Brien, T. J. 2007a, MNRAS, 382, 1233

Wareing, C. J., Zijlstra, A. A., \& O’Brien, T. J. 2007b, ApJ, 660, L129

Wilkin, F. P. 1996, ApJ, 459, L31

Wright, E. L., Eisenhardt, P. R. M., Mainzer, A. K., et al. 2010, AJ, 140, 1868 